\title{
The oxytocin paradox
}

\author{
Richard A. I. Bethlehem ${ }^{1 *}$, Simon Baron-Cohen ${ }^{1,2}$, Jack van Honk ${ }^{3,4}$, Bonnie Auyeung ${ }^{1,5}$ and \\ Peter A. Bos ${ }^{3,4}$ \\ 1 Department of Psychiatry, Autism Research Centre, University of Cambridge, Cambridge, UK \\ ${ }^{2}$ Cambridgeshire and Peterborough NHS Foundation Trust, CLASS Clinic, Cambridge, UK \\ ${ }^{3}$ Department of Experimental Psychology, Utrecht University, Utrecht, Netherlands \\ ${ }^{4}$ Department of Psychiatry and Mental Health, J-Block Groote Schuur Hospital Observatory, University of Cape Town, Cape Town, South Africa \\ ${ }^{5}$ Psychology Department, Edinburgh University, Edinburgh, UK \\ ${ }^{*}$ Correspondence: rb643@cam.ac.uk
}

Edited by:

Elissar Andari, Emory University, USA

Reviewed by:

Jorge Moll, D'Or Institute for Research and Education (IDOR), Brazil

Elissar Andari, Emory University, USA

James P. Burkett, Emory University, USA

Keywords: oxytocin, reward processing, anxiety, autism spectrum disorder, translational research, reward

In 2005, Kosfeld et al. published their now seminal paper showing that intranasal oxytocin (OXT) administration increased interpersonal trust (Kosfeld et al., 2005). This finding spawned broad interest into the effects of OXT on social and emotional behavior in humans (Bos et al., 2012), and its implications for translational medicine (Meyer-Lindenberg et al., 2011; Striepens et al., 2011). Over the years OXT has gained the reputation of facilitating empathy and affiliation, based on early findings reporting beneficial effects of OXT on trust (Kosfeld et al., 2005), social support (Heinrichs et al., 2003), and processing social information (Hollander et al., 2007; Savaskan et al., 2008; Unkelbach et al., 2008; Hurlemann et al., 2010). This view is supported by studies showing that OXT improves cognitive empathic abilities such as mindreading (Domes et al., 2007; Bartz et al., 2010; Guastella et al., 2010) and recognizing positive emotional expressions (Marsh et al., 2010). As a result of these positive effects on social behavior, there has been considerable speculation about OXT's therapeutic potential in people with social and emotional disabilities.

This prosocial view of OXT has been challenged by findings showing that the effects of OXT are strongly contextdependent (Bartz et al., 2011; Bos et al., 2012). For example, OXT has also been shown to increase envy and gloating (Shamay-Tsoory et al., 2009), defensiveness toward out-group members (De Dreu et al., 2010, 2011) and increased in-group conformity (Stallen et al., 2012). Although this ethnocentrism might be considered prosocial within one's own group, defensiveness toward an out-group is not, and extreme ethnocentrism often leads to nationalism or even racism.

This paradox gives rise to two questions. First, how can the beneficial effects of OXT on empathy, trust and affiliation be compatible with its seemingly contradictory anti-social effects? Second, what implication does this have for the therapeutic potential of OXT in social and emotional neurodevelopmental conditions? With regard to the first question, we provide a brief overview of the literature aiming to understand the mechanism(s) underlying OXT's efficacy. There are two main perspectives on how OXT affects social behavior (via anxiety reduction or increasing social salience), which try to reconcile its pro- and anti-social roles, but there have been no attempts to date to integrate these into a single viewpoint. A potential third factor, reward sensitivity might aid this integration. We propose a model that unites these perspectives, providing new avenues of research into OXT's efficacy in social and emotional neurodevelopmental conditions.

\section{MECHANISMS UNDERLYING OXT's EFFECT ON SOCIAL BEHAVIOR}

Early attempts to reconcile different effects of OXT on social behavior suggested interaction effects between stress, anxiety and social approach (Heinrichs and Domes, 2008; Heinrichs et al., 2009). An excellent review (Churchland and
Winkielman, 2012) highlights OXT's anxiolytic effects as a key mechanism. Churchland and Winkielman (2012) argue that low-level anxiety drives higher-order social-cognitive effects. A large body of animal literature also supports the idea of OXT as an anxiolytic (Bale et al., 2001; Ring et al., 2006; Ebitz et al., 2013). Ebitz and colleagues showed that OXT reduces social vigilance in the rhesus macaque (Ebitz et al., 2013), potentially by reducing social threat or anxiety. In humans, a reduction in anxiety could facilitate "pro-social" behavior by eliminating social threat during social interactions. Consistent with this, there is evidence for anxiolytic effects of OXT in humans with anxiety disorder (Labuschagne et al., 2010). Labuschagne et al. (2010) showed that OXT decreases amygdala activity in response to fearful faces in people with a generalized social anxiety disorder (GSAD), to the level of typical controls.

Decreased social threat following OXT administration is also in line with findings showing improved coping mechanisms in stressful situations (Heinrichs et al., 2003). Although there is broad consensus about the anxiolytic properties of OXT, its antisocial effects are not entirely explained by this. As mentioned above, earlier findings (De Dreu et al., 2010, 2011) demonstrated increased inout-group differences after OXT administration (but see Shamay-Tsoory et al., 2013). However, reduced anxiety would be expected to lead to decreased in- and out-group differences by also reducing 
anxiety toward out-group members. In addition, direct empirical tests of the anxiolytic effects of OXT in humans has shown that oxytocin can increase anxiety toward an unpredictable threat (Grillon et al., 2012). This suggests that OXT's anxiolytic properties cannot completely account for the differences in behavioral findings.

Another perspective on the effects of OXT on social behavior involves social salience. It has been hypothesized that OXT increases sensitivity for salient social cues (Bartz et al., 2011; Striepens et al., 2012; Wittfoth-Schardt et al., 2012). This is known as the "social salience hypothesis" and fits with findings of improved mind-reading and increased eye contact (Domes et al., 2007; Guastella et al., 2008). Increasing the salience of subtle contextual eye-cues could indeed improve mind-reading and might cause an attentional bias toward such cues. However, the effects of OXT administration on promoting ethnocentrism emerge, regardless of the valence of an intergroup comparison (De Dreu et al., 2010, 2011). This suggests that these effects cannot be explained by the notion that OXT administration generally increases perceptual salience for social cues, as that would alter valence ratings. Interestingly, recent work has demonstrated that affiliative emotion (a primary target in OXT research) may be distinguishable from general emotional valence (Moll et al., 2012). In addition, Striepens et al. (2012) showed that OXT administration did not alter valence ratings for aversive social stimuli. Since the administration of OXT also facilitated startle response and memory for negative cues, these authors conclude that OXT administration may promote general "approach and protective behavior, but with heightened caution" (Striepens et al., 2012). It is likely that OXT does affect affiliative emotion but it is possible that, depending on the context, general emotional valence is not affected. How OXT does this remains an open question. It seems that, by itself, the social salience hypothesis also cannot fully explain the varied behavioral findings.

In addition to being an anxiolytic and potentially increasing perceptual salience of social cues, OXT may exert its effects on social behavior via a third mechanism: increased reward sensitivity (Leckman,
2011; Strathearn, 2011; Dolen et al., 2013). The idea that reward sensitivity is an important contributing factor to OXT's effect on social behavior is not new (Young et al., 2001), nor necessarily incompatible with the social salience hypothesis (Weisman and Feldman, 2013). Animal studies have shown strong links between OXT and the reward circuitry, specifically in the ventral striatum (Shahrokh et al., 2010; D'Cunha et al., 2011; Keebaugh and Young, 2011; Baracz and Cornish, 2013). A recent study also demonstrated involvement of reward circuits in the efficacy of OXT in humans (Groppe et al., 2013). Groppe et al. (2013) showed that the modulation of social salience by OXT takes place in the human ventral tegmental area (a key area in the mesocorticolimbic dopamine-system), confirming previous animal findings (Shahrokh et al., 2010). Similarly, Strathearn et al. (2009) showed that the ventral striatum is activated during mother-infant interaction, which is accompanied by increased peripheral OXT release.

Two neurobiological pathways may underlie OXT's effect on reward processing: an OXT-dopamine (Shahrokh et al., 2010; Baracz and Cornish, 2013) pathway and an OXT-opioid pathway (Gu and Yu, 2007). The former likely influences the "wanting" aspect of reward processing, whereas the latter likely pertains to the "liking" aspect of reward processing (Berridge et al., 2009). The opioid-OXT pathway is also likely to be involved in OXT's effects on social bonding and affiliation (Burkett and Young, 2012). If either type of reward processing is affected by OXT it is possible that this will also affect reward learning (Berridge et al., 2009).

Lastly, OXT has also been repeatedly associated with drugs of abuse and addiction (Burkett and Young, 2012; Carson et al., 2013; Sarnyai and Kovacs, 2013), which also act on the reward system. It has even been suggested that OXT's facilitation of social reward sensitivity may somewhat override that of drug-induced reward (Sarnyai and Kovacs, 2013; Tops et al., 2013). The way in which reward sensitivity precisely affects social interactions remains unknown, but these findings suggest that OXT influences the means by which social reward sensitivity modulates social behavior. Additionally, context may play a role in the exact type of pathway affected.

\section{FUTURE DIRECTIONS}

The three different mechanisms reviewed here are not mutually exclusive. For example, decreased anxiety could lead to increased sensitivity for social salience, and vice versa. Together, anxiety and social reward sensitivity influence attribution of social salience. They might, in a given context, either promote or impede social behavior. We hypothesize that the mechanism by which OXT affects social behavior depends upon these two systems (see Figure 1). Subsequently, the environment provides feedback to the OXT system about the resulting social behavior, influencing anxiety and reward sensitivity. This synergy in turn determines the salience of social cues in a particular context.

In people with autism spectrum conditions (ASC) or GSAD this system might be disturbed, resulting in social interaction deficits. Recent research has shown hypoactivation in the reward circuitry in ASC during social reward processing (Delmonte et al., 2012). In addition, people with ASC show increased anxiety (White et al., 2009), which might lead to an attentional bias toward negative cues (Winton et al., 1995) in social interaction. Together, these factors create a negative feedback loop, which further enhances this negative spiral. In such situations OXT may have a positive effect by reducing anxiety and increasing the rewarding value of social interactions. The social motivation hypothesis (SMH) for ASC (Stavropoulos and Carver, 2013) indeed suggests that reward sensitivity is decreased in ASC and that OXT helps alleviate this. For example, it has been shown that OXT administration leads people with ASC to respond more appropriately to reciprocity in a cyberball game (Andari et al., 2010). Increased reward sensitivity would thus enable participants to better distinguish which player offers the most positive interaction. Furthermore, reduced anxiety for social interaction (such as direct eye contact) may modulate gazetime (Domes et al., 2013d), and increased reward sensitivity may shift attention toward positive social cues (Domes et al., 2013c). In individuals with ASC, this could modulate face and emotion processing 


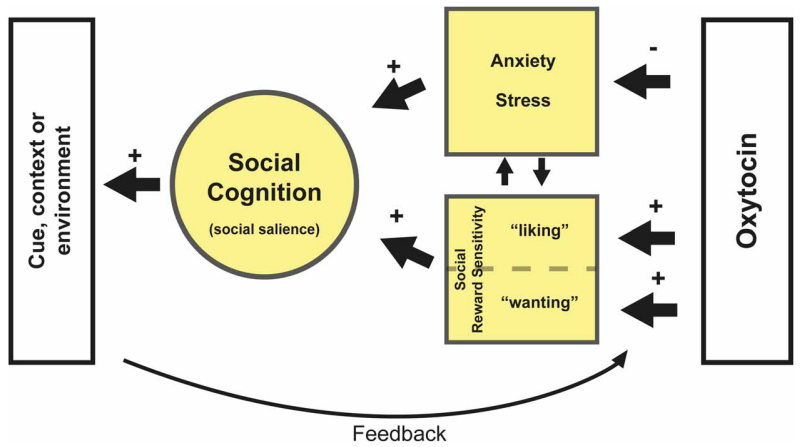

FIGURE 1 | Oxytocin reduces anxiety and stress for social interaction and increases social reward sensitivity. With respect to the latter, OXT likely affects hedonistic reward processing (e.g., "liking") via its interaction with the opioid system and incentive reward processing (e.g., "wanting") via a striatal-dopamine pathway (Berridge et al., 2009). Which pathway is affected likely depends on the specific context. Both reduced anxiety and increased reward sensitivity might increase sensitivity for social salience but also directly improve aspects of social cognition depending on person and context. Furthermore the subsequent contextual or environmental feedback affects the sensitivity and plasticity of the OXT system depending on its valence (e.g., positive or negative feedback). Positive feedback after OXT administration, such as pleasant social interaction, might reinforce the sensitivity for social reward and further decrease anxiety. This feedback potentially also alters the plasticity of the OXT system. For example, more OXT may be released during social interaction. Another potential scenario is that OXT administration leads to decreased anxiety, which in turn leads to heightened social salience. Which may also involve paying more attention to (potentially) negative social cues. Administration of OXT might therefore have a stronger impact on this negative feedback in neurotypical individuals, as was shown by Striepens et al. (2012). Lastly, any type of feedback resulting from altered social reward processing is likely to affect reward learning.

(Domes et al., 2013a,b). Reducing anxiety and improving the reward value of social interaction would also improve emotion recognition and social behavior in individuals with and without ASC (Hollander et al., 2007; Guastella et al., 2010; Domes et al., 2013b). Although the anxiolytic effects of OXT have been widely researched, its combination with reward sensitivity may prove to be a novel target for OXT administration in ASC. Interestingly, this might also help explain the variation of the effects of OXT seen across the population.

In individuals that do not experience heightened anxiety in social situations or experience reduced reward sensitivity, OXT might not necessarily show beneficial effects. Moreover, it might even impede social behavior. For example, by decreasing anxiety social vigilance might become blunted (Ebitz et al., 2013), while increasing reward sensitivity could possibly lead to gloating (Shamay-Tsoory et al., 2009). Furthermore, ethnocentrism occurs naturally in the normal population (Yzerbyt and Demoulin, 2010): under normal circumstances people tend to favor their own group over out-group members. Thus, interaction with in-group members is generally considered more rewarding. Also, a negative interaction with out-group members may constitute a form of negative feedback compared to positive interaction with in-group members. Our model predicts that administration of OXT would increase these differences. It strengthens the rewarding type of interaction and reinforces the effect of negative feedback. In this context, OXT indeed promotes ethnocentrism (De Dreu et al., 2010, 2011).

In conclusion, over recent years it has become clear that the effects of OXT administration on human social behavior are not best framed in absolute terms such as pro- or antisocial. We aim to shift the focus to underlying core processes such as anxiety and reward sensitivity. When applied to studied contexts, the modulation of OXT on these core processes can lead to different behavioral outcomes dependent on person and situation, especially since reward processing and anxiety in specific contexts differs from person to person. More research is needed to investigate how the dynamics of OXT modulation on these core processes translates to different contexts and groups.

\section{ACKNOWLEDGMENTS}

Richard A. I. Bethlehem was funded by the MRC UK, the Pinsent Darwin Trust and the Cambridge Home and EU Scholarship Scheme (CHESS). Simon Baron-Cohen and Bonnie Auyeung were supported by the MRC UK, the Wellcome Trust, and the Autism Research Trust, during the period of this work. Simon Baron-Cohen is part of the NIHR CLAHRC for Cambridgeshire and Peterborough NHS Foundation Trust. Jack van Honk and Peter A. Bos were supported for their work on this project by the Netherlands Society of Scientific Research: \#056-24-010.

\section{REFERENCES}

Andari, E., Duhamel, J. R., Zalla, T., Herbrecht, E., Leboyer, M., and Sirigu, A. (2010). Promoting social behavior with oxytocin in high-functioning autism spectrum disorders. Proc. Natl. Acad. Sci. U.S.A. 107, 4389-4394. doi: 10.1073/pnas.0910249107

Bale, T. L., Davis, A. M., Auger, A. P., Dorsa, D. M., and Mccarthy, M. M. (2001). CNS region-specific oxytocin receptor expression: importance in regulation of anxiety and sex behavior. J. Neurosci. 21, 2546-2552.

Baracz, S. J., and Cornish, J. L. (2013). Oxytocin modulates dopamine-mediated reward in the rat subthalamic nucleus. Horm. Behav. 63, 370-375. doi: 10.1016/j.yhbeh.2012.12.003

Bartz, J. A., Zaki, J., Bolger, N., Hollander, E., Ludwig, N. N., Kolevzon, A., et al. (2010). Oxytocin selectively improves empathic accuracy. Psychol. Sci. 21, 1426-1428. doi: 10.1177/09567976103 83439

Bartz, J. A., Zaki, J., Bolger, N., and Ochsner, K. N. (2011). Social effects of oxytocin in humans: context and person matter. Trends Cogn. Sci. 15, 301-309. doi: 10.1016/j.tics.2011.05.002

Berridge, K. C., Robinson, T. E., and Aldridge, J. W. (2009). Dissecting components of reward: 'liking', 'wanting', and learning. Curr. Opin. Pharmacol. 9, 65-73. doi: 10.1016/j.coph.2008.12.014

Bos, P. A., Panksepp, J., Bluthe, R. M., and Honk, J. V. (2012). Acute effects of steroid hormones and neuropeptides on human socialemotional behavior: a review of single administration studies. Front. Neuroendocrinol. 33:17-35. doi: 10.1016/j.yfrne.2011.01.002

Burkett, J. P., and Young, L. J. (2012). The behavioral, anatomical and pharmacological parallels between social attachment, love and addiction. Psychopharmacology 224, 1-26. doi: 10.1007/s00213-012-2794-x

Carson, D. S., Guastella, A. J., Taylor, E. R., and Mcgregor, I. S. (2013). A brief history of oxytocin and its role in modulating psychostimulant effects. J. Psychopharmacol. 27, 231-247. doi: $10.1177 / 0269881112473788$

Churchland, P. S., and Winkielman, P. (2012). Modulating social behavior with oxytocin: how does it work? What does it mean? Horm. Behav. 61, 392-399. doi: 10.1016/j.yhbeh.2011.12.003 
D'Cunha, T. M., King, S. J., Fleming, A. S., and Levy, F. (2011). Oxytocin receptors in the nucleus accumbens shell are involved in the consolidation of maternal memory in postpartum rats. Horm. Behav. 59, 14-21. doi: 10.1016/j.yhbeh.2010.09.007

De Dreu, C. K., Greer, L. L., Handgraaf, M. J., Shalvi, S., Van Kleef, G. A., Baas, M., et al. (2010). The neuropeptide oxytocin regulates parochial altruism in intergroup conflict among humans. Science 328, 1408-1411. doi: 10.1126/science. 1189047

De Dreu, C. K., Greer, L. L., Van Kleef, G. A., Shalvi, S., and Handgraaf, M. J. (2011). Oxytocin promotes human ethnocentrism. Proc. Natl. Acad. Sci. U.S.A. 108, 1262-1266. doi: 10.1073/pnas.1015316108

Delmonte, S., Balsters, J. H., Mcgrath, J., Fitzgerald, J., Brennan, S., Fagan, A. J., et al. (2012). Social and monetary reward processing in autism spectrum disorders. Mol. Autism 3, 7. doi: 10.1186/20402392-3-7

Dolen, G., Darvishzadeh, A., Huang, K. W., and Malenka, R. C. (2013). Social reward requires coordinated activity of nucleus accumbens oxytocin and serotonin. Nature 501, 179-184. doi: 10.1038 /nature 12518

Domes, G., Heinrichs, M., Kumbier, E., Grossmann, A., Hauenstein, K., and Herpertz, S. C. (2013a). Effects of intranasal oxytocin on the neural basis of face processing in autism spectrum disorder. Biol. Psychiatry 74, 164-171. doi: 10.1016/j.biopsych.2013.02.007

Domes, G., Heinrichs, M., Michel, A., Berger, C., and Herpertz, S. C. (2007). Oxytocin improves "mindreading" in humans. Biol. Psychiatry 61, 731-733. doi: 10.1016/j.biopsych.2006.07.015

Domes, G., Kumbier, E., Heinrichs, M., and Herpertz, S. C. (2013b). Oxytocin promotes facial emotion recognition and amygdala reactivity in adults with asperger syndrome. Neuropsychopharmacology 39, 698-706. doi: 10.1038/npp.2013.254

Domes, G., Sibold, M., Schulze, L., Lischke, A., Herpertz, S. C., and Heinrichs, M. (2013c). Intranasal oxytocin increases covert attention to positive social cues. Psychol. Med. 43, 1747-1753. doi: 10.1017/S0033291712002565

Domes, G., Steiner, A., Porges, S. W., and Heinrichs, M. (2013d). Oxytocin differentially modulates eye gaze to naturalistic social signals of happiness and anger. Psychoneuroendocrinology 38, 1198-1202. doi: 10.1016/j.psyneuen.2012. 10.002

Ebitz, R. B., Watson, K. K., and Platt, M. L. (2013). Oxytocin blunts social vigilance in the rhesus macaque. Proc. Natl. Acad. Sci. U.S.A. 110, 11630-11635. doi: 10.1073/pnas.1305230110

Grillon, C., Krimsky, M., Charney, D. R., Vytal, K., Ernst, M., and Cornwell, B. (2012). Oxytocin increases anxiety to unpredictable threat. $\mathrm{Mol}$. Psychiatry 18, 958-960. doi: 10.1038/mp.2012.156

Groppe, S. E., Gossen, A., Rademacher, L., Hahn, A., Westphal, L., Gründer, G., et al. (2013). Oxytocin influences processing of socially relevant cues in the ventral tegmental area of the human brain. Biol. Psychiatry 74, 172-179. doi: 10.1016/j. biopsych.2012.12.023

$\mathrm{Gu}, \mathrm{X}$. L., and Yu, L. C. (2007). Involvement of opioid receptors in oxytocin-induced antinociception in the nucleus accumbens of rats. J. Pain 8, 85-90. doi: 10.1016/j.jpain.2006.07.001
Guastella, A. J., Einfeld, S. L., Gray, K. M., Rinehart, N. J., Tonge, B. J., Lambert, T. J., et al. (2010). Intranasal oxytocin improves emotion recognition for youth with autism spectrum disorders. Biol. Psychiatry 67, 692-694. doi: 10.1016/j.biopsych.2009.09.020

Guastella, A. J., Mitchell, P. B., and Dadds, M. R. (2008). Oxytocin increases gaze to the eye region of human faces. Biol. Psychiatry 63, 3-5. doi: 10.1016/j.biopsych.2007.06.026

Heinrichs, M., Baumgartner, T., Kirschbaum, C., and Ehlert, U. (2003). Social support and oxytocin interact to suppress cortisol and subjective responses to psychosocial stress. Biol. Psychiatry 54, 1389-1398. doi: 10.1016/S00063223(03)00465-7

Heinrichs, M., and Domes, G. (2008). Neuropeptides and social behaviour: effects of oxytocin and vasopressin in humans. Prog. Brain Res. 170, 337-350. doi: 10.1016/S0079-6123(08)00428-7

Heinrichs, M., Von Dawans, B., and Domes, G. (2009). Oxytocin, vasopressin, and human social behavior. Front. Neuroendocrinol. 30:548-557. doi: 10.1016/i.yfrne.2009.05.005

Hollander, E., Bartz, J., Chaplin, W., Phillips, A., Sumner, J., Soorya, L., et al. (2007). Oxytocin increases retention of social cognition in autism. Biol. Psychiatry 61, 498-503. doi: 10.1016/j.biopsych.2006.05.030

Hurlemann, R., Patin, A., Onur, O. A., Cohen, M. X., Baumgartner, T., Metzler, S., et al. (2010). Oxytocin enhances amygdala-dependent, socially reinforced learning and emotional empathy in humans. J. Neurosci. 30, 4999-5007. doi: 10.1523/JNEUROSCI.5538-09.2010

Keebaugh, A. C., and Young, L. J. (2011). Increasing oxytocin receptor expression in the nucleus accumbens of pre-pubertal female prairie voles enhances alloparental responsiveness and partner preference formation as adults. Horm. Behav. 60, 498-504. doi: 10.1016/j.yhbeh.2011.07.018

Kosfeld, M., Heinrichs, M., Zak, P. J., Fischbacher, U., and Fehr, E. (2005). Oxytocin increases trust in humans. Nature 435, 673-676. doi: 10.1038 /nature03701

Labuschagne, I., Phan, K. L., Wood, A., Angstadt, M., Chua, P., Heinrichs, M., et al. (2010). Oxytocin attenuates amygdala reactivity to fear in generalized social anxiety disorder. Neuropsychopharmacology 35, 2403-2413. doi: $10.1038 /$ npp. 2010.123

Leckman, J. F. (2011). Variations in maternal behavior-oxytocin and reward pathways-peripheral measures matter?! Neuropsychopharmacology 36, 2587-2588. doi: 10.1038/npp.2011.201

Marsh, A. A., Yu, H. H., Pine, D. S., and Blair, R. J. (2010). Oxytocin improves specific recognition of positive facial expressions. Psychopharmacology 209, 225-232. doi: 10.1007/s00213-010-1780-4

Meyer-Lindenberg, A., Domes, G., Kirsch, P., and Heinrichs, M. (2011). Oxytocin and vasopressin in the human brain: social neuropeptides for translational medicine. Nat. Rev. Neurosci. 12, 524-538. doi: 10.1038/nrn3044

Moll, J., Bado, P., De Oliveira-Souza, R., Bramati, I. E., Lima, D. O., Paiva, F. F., et al. (2012). A neural signature of affiliative emotion in the human septohypothalamic area. J. Neurosci. 32,
12499-12505. doi: 10.1523/JNEUROSCI.6508-11. 2012

Ring, R. H., Malberg, J. E., Potestio, L., Ping, J., Boikess, S., Luo, B., et al. (2006). Anxiolytic-like activity of oxytocin in male mice: behavioral and autonomic evidence, therapeutic implications. Psychopharmacology 185, 218-225. doi: 10.1007/s00213-005-0293-z

Sarnyai, Z., and Kovacs, G. L. (2013). Oxytocin in learning and addiction: from early discoveries to the present. Pharmacol. Biochem. Behav. doi: 10.1016/j.pbb.2013.11.019. [Epub ahead of print].

Savaskan, E., Ehrhardt, R., Schulz, A., Walter, M., and Schachinger, H. (2008). Post-learning intranasal oxytocin modulates human memory for facial identity. Psychoneuroendocrinology 33, 368-374. doi: 10.1016/j.psyneuen.2007.12.004

Shahrokh, D. K., Zhang, T. Y., Diorio, J., Gratton, A., and Meaney, M. J. (2010). Oxytocin-dopamine interactions mediate variations in maternal behavior in the rat. Endocrinology 151, 2276-2286. doi: 10.1210/en.2009-1271

Shamay-Tsoory, S. G., Abu-Akel, A., Palgi, S., Sulieman, R., Fischer-Shofty, M., Levkovitz, Y., et al. (2013). Giving peace a chance: oxytocin increases empathy to pain in the context of the Israeli-Palestinian conflict. Psychoneuroendocrinology 38, 3139-3144. doi: 10.1016/j.psyneuen.2013.09.015

Shamay-Tsoory, S. G., Fischer, M., Dvash, J., Harari, H., Perach-Bloom, N., and Levkovitz, Y. (2009). Intranasal administration of oxytocin increases envy and schadenfreude (gloating). Biol. Psychiatry 66, 864-870. doi: 10.1016/j.biopsych.2009. 06.009

Stallen, M., De Dreu, C. K., Shalvi, S., Smidts, A., and Sanfey, A. G. (2012). The herding hormone: oxytocin stimulates in-group conformity. Psychol. Sci. 23, 1288-1292. doi: 10.1177/0956797612 446026

Stavropoulos, K. K., and Carver, L. J. (2013). Research review: social motivation and oxytocin in autism-implications for joint attention development and intervention. J. Child Psychol. Psychiatry 54, 603-618. doi: 10.1111/jcpp.12061

Strathearn, L. (2011). Maternal neglect: oxytocin, dopamine and the neurobiology of attachment. J. Neuroendocrinol. 23, 1054-1065. doi: 10.1111/j.1365-2826.2011.02228.x

Strathearn, L., Fonagy, P., Amico, J., and Montague, P. R. (2009). Adult attachment predicts maternal brain and oxytocin response to infant cues. Neuropsychopharmacology 34, 2655-2666. doi: 10.1038/npp.2009.103

Striepens, N., Kendrick, K. M., Maier, W., and Hurlemann, R. (2011). Prosocial effects of oxytocin and clinical evidence for its therapeutic potential. Front. Neuroendocrinol. 32:426-450. doi: 10.1016/j.yfrne.2011.07.001

Striepens, N., Scheele, D., Kendrick, K. M., Becker, B., Schafer, L., Schwalba, K., et al. (2012). Oxytocin facilitates protective responses to aversive social stimuli in males. Proc. Natl. Acad. Sci. U.S.A. 109, 18144-18149. doi: 10.1073/pnas.1208852109

Tops, M., Koole, S. L., Ijzerman, H., and BuismanPijlman, F. T. (2013). Why social attachment and oxytocin protect against addiction and stress: Insights from the dynamics between ventral and dorsal corticostriatal systems. Pharmacol. 
Biochem. Behav. doi: 10.1016/j.pbb.2013.07.015. [Epub ahead of print].

Unkelbach, C., Guastella, A. J., and Forgas, J. P. (2008). Oxytocin selectively facilitates recognition of positive sex and relationship words. Psychol. Sci. 19, 1092-1094. doi: 10.1111/j.14679280.2008.02206.x

Weisman, O., and Feldman, R. (2013). Oxytocin effects on the human brain: findings, questions, and future directions. Biol. Psychiatry 74, 158-159. doi: 10.1016/j.biopsych.2013.05.026

White, S. W., Oswald, D., Ollendick, T., and Scahill, L. (2009). Anxiety in children and adolescents with autism spectrum disorders. Clin. Psychol. Rev. 29, 216-229. doi: 10.1016/j.cpr.2009. 01.003

Winton, E. C., Clark, D. M., and Edelmann, R. J. (1995). Social anxiety, fear of negative evaluation and the detection of negative emotion in others. Behav. Res. Ther. 33, 193-196. doi: 10.1016/00057967(94)E0019-F

Wittfoth-Schardt, D., Grunding, J., Wittfoth, M., Lanfermann, H., Heinrichs, M., Domes, G., et al. (2012). Oxytocin modulates neural reactivity to children's faces as a function of social salience. Neuropsychopharmacology 37, 1799-1807. doi: 10.1038/npp.2012.47

Young, L. J., Lim, M. M., Gingrich, B., and Insel, T. R. (2001). Cellular mechanisms of social attachment. Horm. Behav. 40, 133-138. doi: 10.1006/hbeh.2001.1691

Yzerbyt, V., and Demoulin, S. (2010). "Intergroup relations," in Handbook of Social Psychology, 5th Edn, eds S. T. Fiske, D. T. Gilbert, and G. Lindzey (New York, NY: Wiley), 1024-1083.
Received: 06 December 2013; accepted: 30 January 2014; published online: 17 February 2014.

Citation: Bethlehem RAI, Baron-Cohen S, van Honk J, Auyeung B and Bos PA (2014) The oxytocin paradox. Front. Behav. Neurosci. 8:48. doi: 10.3389/fnbeh. 2014.00048

This article was submitted to the journal Frontiers in Behavioral Neuroscience.

Copyright () 2014 Bethlehem, Baron-Cohen, van Honk, Auyeung and Bos. This is an open-access article distributed under the terms of the Creative Commons Attribution License (CC BY). The use, distribution or reproduction in other forums is permitted, provided the original author(s) or licensor are credited and that the original publication in this journal is cited, in accordance with accepted academic practice. No use, distribution or reproduction is permitted which does not comply with these terms. 